\title{
A HOMOGENEOUS DIFFERENTIAL SYSTEM OF INFINITE ORDER WITH NONVANISHING SOLUTION
}

\author{
S. BOCHNER AND D. V. WIDDER
}

1. Introduction. The only solution of the homogeneous differential system

$$
\begin{gathered}
\frac{d^{2 k-1}}{d t^{2 k-1}}\left[t^{k} f(t)\right]=0, \\
f(0+)=f(+\infty)=0
\end{gathered}
$$

is $f(t) \equiv 0$. This may be seen by observing that none of the fundamental solutions of equation (1), $t^{n}(n=-k,-k+1, \cdots, k-2)$, satisfies the boundary conditions (2). We now replace equation (1) by another of infinite order, retaining the boundary conditions (2), and investigate the possibility of a nonvanishing solution. We let $k$ become infinite in equation (1) after introduction of a factor. Set

$$
L_{k, t}[f(x)]=\frac{(-t)^{k-1}}{k !(k-2) !} \frac{d^{2 k-1}}{d t^{2 k-1}}\left[t^{k} f(t)\right]
$$

and consider the system

$$
\lim _{x \rightarrow \infty} L_{k, t}[f(x)]=0, \quad 0<t<\infty,
$$

with the boundary conditions (2). Since the differential operator (3) serves to invert the Stieltjes transform, ${ }^{1}$

$$
\begin{aligned}
& f(x)=\int_{0}^{\infty} \frac{\phi(t)}{x+t} d t, \\
& \lim _{x \rightarrow \infty} L_{k, t}[f(x)]=\phi(t),
\end{aligned}
$$

it is clear that the only solution of the system (4), (2) of the form (5) must vanish identically. Accordingly if the limit (4) exists boundedly, ${ }^{2}$ for example, there is no nonvanishing solution of the system. However, we shall show that if the limit (4) merely exists at each point of the interval then there are many nonvanishing solutions of the system. One very simple solution is $f(t)=t(1+t)^{-2}$. We shall find

Presented to the Society, September 4,1947; received by the editors June 25, 1947. 345.

${ }^{1}$ See The Laplace transform by D. V. Widder, Princeton University Press, 1946, p.

2 D. V. Widder, loc. cit. p. 373. 
it convenient to make an exponential change of variable which will change equation (4) into

$$
\lim _{n \rightarrow \infty} D \prod_{k=1}^{n}\left(1-\frac{D^{2}}{k^{2}}\right) y(x)=0
$$

where $D$ indicates differentiation with respect to $x$. The system in question becomes

$$
\begin{gathered}
(\sin \pi D) y(x)=0, \\
y(-\infty)=y(+\infty)=0,
\end{gathered}
$$

and a nonvanishing solution is

$$
y(x)=e^{-x}\left(1+e^{-x}\right)^{-2} .
$$

\section{Existence of a nonvanishing solution.}

THEOREM 1. The function

$$
h(x)=\frac{e^{-x}}{\left(1+e^{-x}\right)^{2}}
$$

satisfies the system (6), (7).

To prove this let us introduce the following notation:

$$
\begin{aligned}
E_{n}(D) & =D \prod_{k=1}^{n}\left(1-\frac{D^{2}}{k^{2}}\right) \\
g(x) & =\frac{1}{1+e^{-x}}, \\
c_{n} & =\frac{(2 n+1) !}{n ! n !}
\end{aligned}
$$

We shall show by induction that

$$
E_{n}(D) g(x)=c_{n}[h(x)]^{n+1}
$$

This is true for $n=0$ since $g^{\prime}(x)=h(x)$. Assume equation (8) true when $n$ is replaced by $n-1$ and differentiate both sides twice with respect to $x$,

$$
\frac{d^{2}}{d x^{2}} E_{n-1}(D) g(x)=-c_{n-1}\left\{\frac{2 n(2 n+1) e^{-(n+1) x}}{\left(1+e^{-x}\right)^{2 n+2}}+\frac{n^{2} e^{-n x}}{\left(1+e^{-x}\right)^{2 n}}\right\} .
$$

If this equation is divided by $n^{2}$ and subtracted from equation (8) for 
$n-1$ we obtain

$$
\begin{aligned}
E_{n}(D) g(x) & =\frac{c_{n-1}}{n^{2}} \frac{2 n(2 n+1) e^{-(n+1) x}}{\left(1+e^{-x}\right)^{2 n+2}} \\
& =c_{n} h(x)^{n+1}
\end{aligned}
$$

By one more differentiation we have

$$
E_{n}(D) h(x)=c_{n}(n+1) h(x)^{n} h^{\prime}(x) .
$$

Since $h(x)$ is an even function it is clear that $E_{n}(D) h(x)$ vanishes at $x=0$. For each $x \neq 0$ the right-hand side of equation (10) is the general term of a convergent series and hence tends to zero with $n$. The test ratio of the series is

$$
\lim _{n \rightarrow \infty} \frac{c_{n}}{c_{n-1}}\left(\frac{n+1}{n}\right) h(x)=4 h(x),
$$

and this is clearly less than unity. Thus

$$
(\sin \pi D) h(x)=0, \quad-\infty<x<\infty .
$$

It is clear by inspection that $h( \pm \infty)=0$. This proves the theorem.

\section{Derivatives of the solution.}

THEOREM 2. Every even derivative of the function $h(x)$ of Theorem 1 satisfies the system (6), (7).

By equation (9) we have $E_{n}(D) g^{(p)}(x)=c_{n} D^{p} h(x)^{n+1}, p=0,1,2, \cdots$. Without computing the $p$ th derivative of $h(x)^{n+1}$ completely we can obtain an upper bound for it as follows. It is clear that

$$
D^{p} h(x)^{n+1}=D^{p} \frac{1}{\left(e^{x / 2}+e^{-x / 2}\right)^{2 n+2}}=\frac{a_{p}(x)}{\left(e^{x / 2}+e^{-x / 2}\right)^{2 n+p+2}},
$$

where

$$
a_{p}(x)=\sum_{j=0}^{p} c_{p, j} e^{(j-p / 2) x}
$$

Here the coefficients $c_{p, j}$ need not be determined. If

$$
\left|c_{p, j}\right| \leqq A_{p} C_{p, j}, \quad j=0,1, \cdots, p,
$$

then

$$
\left|a_{p}(x)\right| \leqq A_{p}\left(e^{x / 2}+e^{-x / 2}\right)^{p}, \quad-\infty<x<\infty
$$


By differentiation of equation (12) we have

$$
\left|a_{p}^{\prime}(x)\right| \leqq A_{p} \frac{p}{2}\left(e^{x / 2}+e^{-x / 2}\right)^{p} .
$$

By induction on $p$ we obtain by differentiating equation (11) that

$$
a_{p+1}(x)=a_{p}^{\prime}(x)\left(e^{x / 2}+e^{-x / 2}\right)-2^{-1}(2 n+p+2) a_{p}(x)\left(e^{x / 2}-e^{-x / 2}\right) .
$$

Then inequalities (13) and (14) give

$$
\left|a_{p+1}(x)\right| \leqq A_{p}(n+p+1)\left(e^{x / 2}+e^{-x / 2}\right)^{p+1}
$$

or

$$
A_{p+1}=(n+p+1) A_{p} .
$$

For $p=0, a_{p}(x)=1$, and $A_{0}=1$. Hence

$$
A_{p}=\frac{(n+p) !}{n !}
$$

and

$$
\begin{aligned}
&\left|D^{p}[h(x)]^{n+1}\right| \leqq \frac{(n+p) !}{n !}[h(x)]^{n+1} \\
&\left|E_{n}(D) h^{(p)}(x)\right| \leqq c_{n} \frac{(n+p+1) !}{n !}[h(x)]^{n+1} .
\end{aligned}
$$

As in $\$ 2$ the right-hand side of inequality (15) tends to zero with $1 / n$ for each $x \neq 0$. Moreover if $p$ is even, the left-hand side is an odd function and consequently vanishes at the origin. Hence $h^{(p)}(x)$ satisfies equation (6) for even $p$.

Since

$$
\begin{aligned}
h^{(p)}(x) & =E_{0}(D) g^{(p)}(x)=c_{0} D^{p}[h(x)], \\
\left|h^{(p)}(x)\right| & \leqq c_{0} p ! h(x),
\end{aligned}
$$

it follows that $h^{(p)}(x)$ vanishes at $\pm \infty$, and the theorem is proved.

4. Further solutions. We can now obtain a very large class of solutions of the system (6), (7).

THEOREM 3. If the function

$$
f(w)=\sum_{k=0}^{\infty} a_{k} w^{k}
$$

is an even function of order one and minimal type and if $h(x)$ is the 
function of Theorem 1, then the function

$$
F(x)=\sum_{k=0}^{\infty} a_{k} h^{(k)}(x)
$$

satisfies the system (6), (7).

Let $\epsilon_{0}, \epsilon_{1}, \cdots$ be a sequence of complex numbers tending to zero. Then the most general function of order one and minimal type takes the form ${ }^{3}$

$$
f(w)=\sum_{k=0}^{\infty} \frac{\left(\epsilon_{k} w\right)^{k}}{k !} w^{k} .
$$

Since $f(w)$ is even, $\epsilon_{k}=0$ for even $k$. Clearly $F(x)$ is also even, and $E_{n}(D) F(x)$ is odd for each $n$, and vanishes at the origin. That is, $F(x)$ satisfies equation (6) at $x=0$.

If $x \neq 0$ we may determine a positive number $\epsilon$ less than unity and such that

$$
4 e^{-x}<\left(1+e^{-x}\right)^{2}(1-\epsilon) .
$$

Now determine an integer $N$ so large that $\left|\epsilon_{k}\right| \leqq \epsilon$ when $k \geqq N$. We have at once that

$$
E_{n}(D) F(x)=\sum_{k=0}^{\infty} \frac{\stackrel{k}{k}_{k}}{k !} E_{n}(D) h^{(k)}(x) .
$$

Inequality (15) is sufficient to establish the uniform convergence of the series (18) and thus to validate the term-by-term differentiation. By Theorem 2 it is clear that

$$
\lim _{n \rightarrow \infty} \sum_{k=0}^{N} \frac{\epsilon_{k}^{k}}{k !} E_{n}(D) h^{(k)}(x)=0 .
$$

Moreover, by inequality (15)

$$
\begin{aligned}
\left|\sum_{k=N}^{\infty} \frac{\epsilon_{k}^{k}}{k !} E_{n}(D) h^{(k)}(x)\right| & \leqq c_{n}[h(x)]^{n+1} \sum_{k=N}^{\infty} \frac{(n+k+1) !}{n !} \frac{\epsilon^{k}}{k !} \\
& \leqq \frac{(n+1) c_{n}}{(1-\epsilon)^{n+2}}[h(x)]^{n+1}
\end{aligned}
$$

But this dominating function is again the general term of a series

\footnotetext{
See, for example, L. Bieberbach, Lehrbuch der Funktionentheorie, vol. 2, 1931, p
} 235. 
with test ratio

$$
\lim _{n \rightarrow \infty} \frac{c_{n+1}}{c_{n}} \frac{h(x)}{1-\epsilon}=\frac{4 h(x)}{1-\epsilon} .
$$

This ratio is less than 1 by (17), so that $F(x)$ satisfies (6) for all $x$.

Finally, from inequality (16)

$$
|F(x)| \leqq c_{0} h(x) \sum_{k=0}^{\infty}\left|\epsilon_{k}\right|^{k},
$$

and $F(x)$ also satisfies equations (7). This completes the proof.

5. A counter-example. We can now show that the result of Theorem 3 is best possible in a certain sense.

THEOREM 4. The word "minimal" in Theorem 3 cannot be replaced by the word "normal".

To prove this it will be sufficient to exhibit an even entire function

$$
f(w)=\sum_{k=0}^{\infty} a_{k} w w^{k}
$$

of order 1 and type $t$ (arbitrarily small) such that the function

$$
F(x)=\sum_{k=0}^{\infty} a_{k} h^{(k)}(x)
$$

will fail to satisfy equation (6) for some $x$. We choose $a_{k}=(i t)^{k} / k$ ! for even $k$ and $a_{k}=0$ for odd $k$. Then $f(w)=\cos (t w)$ is a function with the properties desired. Moreover the function $F(x)$ becomes

$$
F(x)=\frac{h(x+i t)+h(x-i t)}{2}=\operatorname{Re} h(x+i t)
$$

Simple computations give

$$
\begin{aligned}
& -\frac{E_{n}(D) F(x)}{(n+1) c_{n}} \\
& =\operatorname{Re}\left\{\frac{\left(e^{x / 2}-e^{-x / 2}\right) \cos (t / 2)+i\left(e^{x / 2}+e^{-x / 2}\right) \sin (t / 2)}{\left[\left(e^{x / 2}+e^{-x / 2}\right) \cos (t / 2)+i\left(e^{x / 2}-e^{-x / 2}\right) \sin (t / 2)\right]^{2 n+3}}\right\} .
\end{aligned}
$$

If we define an argument $\alpha$ and a modulus $\lambda$ as follows,

$$
\tan \alpha=\frac{e^{x / 2}-e^{-x / 2}}{e^{x / 2}+e^{-x / 2}} \tan \frac{t}{2}, \quad \lambda^{2}=e^{x}+e^{-x}+2 \cos t
$$


equation (19) becomes

$$
\begin{aligned}
- & \frac{\lambda^{2 n+3} E_{n}(D) F(x)}{(n+1) c_{n}} \\
= & \operatorname{Re}\left\{\frac{\left(e^{x / 2}-e^{-x / 2}\right) \cos (t / 2)+i\left(e^{x / 2}+e^{-x / 2}\right) \sin (t / 2)}{e^{i \alpha(2 n+3)}}\right\} .
\end{aligned}
$$

If we choose $x$ so near zero that $\lambda<2$, it is clear that $\lambda^{2 n+8} /\left[(n+1) c_{n}\right]$ approaches zero with $1 / n$. If in addition we choose $x$ so near zero that $\alpha=(2 \pi) / N$, where $N$ is an odd integer (which is seen to be possible from the definition of $\alpha$ ), then the right-hand side of equation (20) reduces to

$$
\left(e^{x / 2}-e^{-x / 2}\right) \cos (t / 2) \neq 0
$$

for infinitely many values of $n$. Hence for such $x$

$$
\lim _{n \rightarrow \infty} E_{n}(D) F(x) \neq 0,
$$

and our result is established.

Princeton University AND

HARVARD UNIVERSITY 